
\title{
3 Research Square \\ Latent tri-lineage potential of human menstrual blood-derived mesenchymal stromal cells revealed by specific in vitro culture conditions
}

\section{Diana Quintero-Espinosa}

Universidad de Antioquía: Universidad de Antioquia

\section{Viviana Soto-Mercado}

Universidad de Antioquía: Universidad de Antioquia

Catherine Quintero-Quinchia

Universidad de Antioquía: Universidad de Antioquia

\section{Miguel Mendivil-Perez}

Universidad de Antioquía: Universidad de Antioquia

\section{Carlos Velez-Pardo}

Universidad de Antioquía: Universidad de Antioquia

Marlene Jimenez-Del-Rio ( $\nabla$ marlene.jimenez@udea.edu.co )

University of Antioquia: Universidad de Antioquia https://orcid.org/0000-0003-3477-2386

\section{Research Article}

Keywords: Menstrual blood, stromal, cholinergic, dopaminergic, astrocyte, calcium, neurogenic

Posted Date: March 9th, 2021

DOI: https://doi.org/10.21203/rs.3.rs-290653/v1

License: (c) (1) This work is licensed under a Creative Commons Attribution 4.0 International License.

Read Full License

Version of Record: A version of this preprint was published at Molecular Neurobiology on July 16th, 2021. See the published version at https://doi.org/10.1007/s12035-021-02442-6. 


\section{Abstract}

Human menstrual blood-derived mesenchymal stromal cells (MenSCs) have become not only an important source of stromal cells for cell therapy but also a cellular source for neurologic disorders in vitro modeling. By using culture protocols originally developed in our laboratory, we show that MenSCs can be converted into floating neurospheres (NSs) using the Fast-N-Spheres medium for 24-72h, and can be transdifferentiated into functional dopaminergic-like (DALNs, 26\% TH+/DAT+ flow cytometry) and cholinergic-like neurons (ChLNs, $46 \%$ ChAT+/VAChT flow cytometry) which responded to dopamine- and acetylcholine- triggered neuronal $\mathrm{Ca} 2+$ inward stimuli when cultured with the NeuroForsk and the Cholinergic-N-Run medium , respectively in a timely fashion (i.e., 4-7 days). Here, we also report a direct transdifferentiation method to induce MenSCs into functional astrocyte-like cells (ALCs) by incubation of MenSCs in commercial Gibco ${ }^{\circledR}$ Astrocyte Medium in 7-days. The MSCs-derived ALCs ( 59\% $\mathrm{GFAP}+/ \mathrm{S} 100 \mathrm{~b}+$ ) were found to respond to glutamate-induced $\mathrm{Ca} 2+$ inward stimuli. Altogether these results show that MenSCs are a reliable source to obtain functional neurogenic cells to further investigate the neurobiology of neurologic disorders.

\section{Introduction}

Mesenchymal stromal cells (MSCs) are multipotent cells with self-renewal ability and differentiating potential [1, 2]. Because of these meaningful features, MSCs hold great promise for the treatment and modeling neurological disorders such as Alzheimer's (AD) and Parkinson's diseases (PD), and for testing potential therapeutic approaches (e.g., [3, 4]). Since MSCs can be obtained from fetal (e.g., umbilical cord Wharton's jelly (UC-WJ), umbilical cord blood, placenta) and adult sources (e.g., dental pulp, gingival tissue, adipocyte tissue, menstrual blood) with minimal technical limitations, minor ethical issues, and/or reduced tumorigenic risk, they have emerged as a useful strategy for regenerative medicine [5]. Accordingly, menstrual-blood stromal cells (hereafter named as MenSCs) have become not only an important source of stromal cells for cell therapy (e.g.,[6, 7]) but also as a cellular source for neurologic disorders in vitro modeling. Indeed, it has been shown that MenSCs can be converted into clonogenic neurosphere-like cells (NSCs) in 10-20 days, which can be transdifferentiated into glial-like and neural-like cells in 12-16 days [8,9]. However, no further data are available to determine whether MenSCs can transdifferentiate into other neuronal lineages such as dopaminergic, cholinergic, or astrocytic neuronal cells [10].

Recently, we have derived MSCs from UC-WJ to obtain neurospheres (NSs) i.e., free-floating spherical cell aggregates, also named as neural precursor cells, in $24 \mathrm{~h}$, and NSs-derived nerve-like cells (NLCs) in 4 days [11]. Moreover, MSCs have also been transdifferentiated into functional cholinergic-like neurons (ChLNs) in 7 days [12]. While UC-WJ-derived MSCs are available for only a minority of individuals who have their samples banked at birth, MenSCs can be isolated with minimal invasiveness and risk to the donor and can be obtained in sufficient numbers to enable expansion in culture. Therefore, MenMSC might represent a fast, safe, and efficient way of generating neural-like cells [4]. 
The present study aimed to investigate whether MenSCs can transdifferentiate into NSs and neuronal lineages with culture protocols originally developed in our laboratory including three culture media such as the Fast-N-Spheres [11], the NeuroForsk [11], and the Cholinergic-N-Run medium [12]. Similar to UC-WJMSCs, we show that MenSCs can be converted into floating NSs using the Fast-N-Spheres medium for 24-72h, and can be transdifferentiated into functional dopaminergic-like neurons (DALNs) and ChLNs which responded to dopamine- and acetylcholine- triggered neuronal $\mathrm{Ca}^{2+}$ inward stimuli when cultured with the NeuroForsk and the Cholinergic-N-Run medium, respectively, in 4-7 days of culture. We also report a direct transdifferentiation method to induce MenSCs into functional astrocyte-like cells (ALCs) by incubation of MenSCs in commercial Gibco ${ }^{\circledR}$ Astrocyte Medium in 7-days. Accordingly, MenSCs-derived ALCs ( 59\% GFAP+/S100b+) were found to respond to glutamate-induced $\mathrm{Ca}^{2+}$ inward stimuli. Altogether these results show that MenSCs are a reliable source to obtain functional neurogenic cells to further investigate the neurobiology of $A D$ and $P D$ neurologic disorders.

\section{Material And Methods}

\section{Isolation and characterization of mesenchymal stromal cells derived from human menstrual blood (MenSCs)}

The menstrual blood samples were collected from three healthy females aged between 18-30 years. Donors provided a signed informed consent approved by the Ethics Committee of the Sede de Investigación Universitaria -SIU-, University of Antioquia, Medellín, Colombia. Menstrual blood (MenB) was collected by cup collection during the first 3 days of menses. Briefly, menstrual blood samples were delivered into the laboratory and mixed with an equal volume of phosphate-buffered saline (PBS) containing $1 \mathrm{mM}$ ethylenediamine tetra-acetic acid (EDTA), with $100 \mathrm{U} / \mathrm{ml}$ penicillin/streptomycin 0.25 $\mathrm{mg} / \mathrm{ml}$ amphotericin B, and subject to cell lysis or standard Ficoll procedures within $24 \mathrm{~h}$ as previously described by Liu et al., 2018 [13]. After centrifugation, the cells suspending in a buffy coat were transferred into a new tube, washed in PBS twice, and suspended in growth medium (low-glucose DMEM medium supplemented with 10\% FBS (Gibco, USA), $100 \mathrm{U} / \mathrm{ml}$ penicillin/streptomycin $0.25 \mathrm{mg} / \mathrm{ml}$ amphotericin $\mathrm{B}$ ) and seeded into $25 \mathrm{~cm}^{2}$ plastic cell culture flasks at $37^{\circ} \mathrm{C}$ with $5 \%$ humidified $\mathrm{CO}_{2}$. The medium was replaced every 3 days leaving behind the adherent cells that were growing as fibroblastic cells in clusters. When the cells reached $80-90 \%$ confluence (P0), the cells were detached by $0.25 \%$ trypsin/1 mM EDTA and sub-cultured to new flasks by the ratio of 1:3. The isolated MenSCs were evaluated for the following characteristics: colony formation capacity (i.e., colonies with a typical adherent growth, colony-forming unit fibroblast activity, and spindle-shaped and fibroblast-like morphology); positivity for the mesenchymal-associated surface markers CD90, CD9, and CD73 (99\% positive); and karyotype with normal shape, number, and distribution. The differentiation capacity into an osteoblast, chondrocyte, and adipocyte lineage of MenSCs, as well as the presence of neuronal precursors and astrocyte markers (e.g., NFL, b-TUB III, GFAP, S100b, TH, ChAT), was assessed according to Mendivil-Perez et al., 2016 and 2019 [12, 14].

\section{Cell differentiation}




\section{Neurospheres (NSs) formation}

MenSCs were seeded at a density of $2.5 \times 10^{4} \mathrm{cells} / \mathrm{cm}^{2}$ in a multi-well plate (Greinner-Bio-one, cat\# 662102) using Fast-N-spheres medium (DMEN F-12 GIBCO®, cat\#11330-032; supplemented with $2 \%$ B27® GIBCO ${ }^{\circledR}$ (cat \#17504-044), $20 \mathrm{ng} / \mathrm{ml}$ basic fibroblast growth factor (bFGF, R\&D Systems, Inc., MN), $20 \mathrm{ng} / \mathrm{ml}$ epidermal growth factor (EGF, Sigma cat\#E9644), $1 \mu \mathrm{g} / \mathrm{ml}$ heparin sodium salt $\AA$, and $100 \mathrm{U} / \mathrm{ml}$ penicillin/streptomycin) for 0,1 and 3 days according to [11].

\section{Astrocyte-like Cells (ALCs) differentiation}

For astrocyte differentiation, $1 \times 10^{3}$ MenSCs / $\mathrm{cm}^{2}$ were seeded in $25 \mathrm{~cm}^{2}$ culture flasks in regular culture medium ( $R C \mathrm{~m}$, DMEM low glucose Sigma cat\# D6046 media supplemented with $10 \% \mathrm{FBS}$ ) until reach $40 \%$ of confluence. Then, the medium was replaced and cells were incubated either in DMEM low glucose media supplemented with $2 \%$ FBS (minimal culture medium, thereafter $\mathrm{MCm}$ ) or Astrocyte medium ${ }^{\circledR}\left(\mathrm{GIBCO}{ }^{\circledR}\right.$, cat\#A1261301) for 0 , 4, and 7 days.

\section{Dopaminergic-like Neurons (DALNs) differentiation}

The MenSCs were seeded at $1 \times 10^{3}$ MenSCs $/ \mathrm{cm}^{2}$ in $25 \mathrm{~cm}^{2}$ culture flasks for $24 \mathrm{~h}$ in regular culture medium (DMEM low glucose media supplemented with 10\% FBS). Then, the medium was removed and cells were incubated either in $\mathrm{MCm}$ or dopaminergic differentiation medium (Neuroforsk medium, DMEM low glucose media supplemented with 2\% FBS Forskolin (Sigma cat\# F6886) $1 \mathrm{mM}$ final concentration) for 0,4 and 7 days according to [11].

\section{Cholinergic-Like Neurons (ChLNs) differentiation}

The MenSCs were seeded at $3-5 \times 10^{3}$ cells $/ \mathrm{cm}^{2}$ in $25 \mathrm{~cm}^{2}$ culture flasks for $24 \mathrm{~h}$ in regular culture medium. Then, the medium was removed and cells were incubated either in $\mathrm{MCm}$ or cholinergic differentiation medium (Cholinergic-N-Run medium containing DMEM/F-12 media 1:1 Nutrient Mixture Gibco (cat\# 10565018, $10 \mathrm{ng} / \mathrm{mL}$ ), basic fibroblast growth factor (bFGF) recombinant human protein (Gibco cat\# 13256029), $50 \mu \mathrm{g} / \mathrm{mL}$ sodium heparine Hep (Sigma-Aldrich cat\# H3393), $0.5 \mu \mathrm{M}$ all-trans retinoic acid, $50 \mathrm{ng} / \mathrm{mL}$ sonic hedgehog peptide (SHH, Sigma cat\# SRP3156) and $1 \% \mathrm{FBS})$ at $37^{\circ} \mathrm{C}$ for 0 , 4 and 7 days.

\section{Western blotting (WB) analysis}

Cells treated with DALNs, ChLNs, NSs, and ALCs differentiation medium for 0,4 , and 7 days were detached with $0.25 \%$ trypsin and lysed in $50 \mathrm{mM}$ Tris- $\mathrm{HCl}, \mathrm{pH} 8.0$, with $150 \mathrm{mM}$ sodium chloride, $1.0 \%$ Igepal CA-630 (NP-40), and 0.1\% sodium dodecyl sulfate and a protease inhibitor cocktail (SigmaAldrich). All lysates were (quantified using the bicinchoninic acid assay; Thermo Scientific cat \# 23225) and $30 \mu \mathrm{g}$ of proteins were loaded onto $12 \%$ electrophoresis gels and transferred onto nitrocellulose membranes (Hybond-ECL, Amersham Biosciences) at $270 \mathrm{~mA}$ for 90 min using an electrophoretic transfer system (BIO-RAD). The membranes were incubated overnight at $4{ }^{\circ} \mathrm{C}$ with monoclonal/ 
polyclonal antibodies against sex-determining region Y)-box 2 (SOX2 cat\# PA1-094, Thermo), anti-Nestin (Thermo, cat \#MA1 5841), dopamine transporter (DAT cat \# PA1-4656), tyrosine hydroxylase (TH, cat\# AB152, Millipore), glial fibrillary acidic protein (GFAP, cat\# sc6170, Santa Cruz), S100ß (cat\# 676604, Biolegend), $\beta$-tubulin III ( $\beta$-TUB III cat\# G712A, Promega), microtubule-associated protein 2 (MAP2, MA125044, Invitrogen), Neurofilament-L (NF-L, cat\# 125044, Thermo), vesicular acetylcholine transporter (VAChT, cat\# SAB4200559, Sigma-Aldrich) and choline acetyltransferase (ChAT, cat\# AB144P, Millipore) primary antibodies (1:5000). Anti-actin antibody (cat \#MAB1501, Millipore; 1:1000) was used as expression control. Secondary infrared antibodies (goat anti-rabbit IRDye ${ }^{\circledR}$ 680RD, cat \#926-68071; donkey anti-goat IRDye ® 680RD, cat \# 926-68074; and goat anti-mouse IRDye ® 800CW, cat \#92632270; LICORBiosciences) 1:1000 was used for Western blotting analysis and data were acquired by using Odyssey software.

\section{Immunofluorescence (IMF) analysis}

For immunofluorescence analysis of neural and astrocytes markers, cells treated with MCm, Neuroforsk, Gibco ${ }^{\circledR}$ Astrocyte Medium (Liao et al., 2016; Mytych et al., 2015; Rao et al., 2015) or Ch-N-Rm medium for 0, 4 and 7 days and Neurospheres Fast medium for 0,24 and 72 hours were fixed with paraformaldehyde for $20 \mathrm{~min}$, followed by Triton X-100 (0.1\%) permeabilization and $5 \%$ bovine serum albumin (BSA) blockage. Cells were then incubated overnight with primary antibodies against DAT, TH, GFAP, S100ß, bTUB III, MAP2, NFL, VAChT, and ChAT proteins (1:500). After exhaustive rinsing, we incubated the cells with secondary fluorescent antibodies (DyLight 488 and 595 donkey anti-rabbit and -goat and -mouse, Cat DI 2488 and DI 1094, respectively) 1:500. The nuclei were stained with Hoechst $33342(1 \mu \mathrm{M}$, life technologies) and images were acquired on a Floyd Cells Imaging Station microscope.

\section{Flow cytometry (FC) analysis of astrocytic, dopaminergic, and cholinergic markers}

Flow cytometry acquisition was used to determine the percentage of GFAP/S100ß, DAT/TH, ChAT/ VAChT double-positive cells, according to previous reports (Moghaddam et al., 2017, Tcw, J et al., 2017, Fathi et al., 2018). Cells treated with MCm, Neuroforsk, astrocyte, or Ch-N-Rm medium at days 0,4 , and 7 were detached with $0.25 \%$ trypsin- EDTA $1 \mathrm{mM}$ and fixed in suspension with paraformaldehyde (overnight). After washing, cells were simultaneously incubated with GFAP, S100ß, DAT, TH, ChAT, and VAChT primary antibodies (1:500) at $4^{\circ} \mathrm{C}$ overnight. Cell suspensions were washed and incubated with DyeLight 594 donkey anti-goat and DyeLight 488 donkey anti-rabbit antibodies (1:500). Finally, cells were washed and re-suspended in PBS for analysis on a Canto cytometer (Beckman coulter). Ten thousand events were acquired and the acquisition analysis was performed using FlowJo 7.6.2 Data Analysis Software. Positive staining was defined as the fluorescence emission that exceeded levels of the population stained with the negative control (only secondary antibodies staining).

\section{Intracellular calcium imaging}

The cytoplasmic $\mathrm{Ca}^{2+}$ concentration $\left(\left[\mathrm{Ca}^{2+}\right]_{\mathrm{i}}\right)$ was measured. Briefly, DALNs, ChLNs, and ALCs cultured in Neuroforsk, Ch-N-Rm, and Astrocyte medium respectively for 0,4 , and 7 days were transferred to a bath 
solution (NBS; in mM: $137 \mathrm{NaCl}, 5 \mathrm{KCl} 2.5 \mathrm{CaCl}_{2}, 1 \mathrm{MgCl}_{2}, 10$ HEPES, pH 7.3, and 22 glucose) containing $\mathrm{a} \mathrm{Ca}^{2+}$ sensitive indicator ( $2 \mu \mathrm{M}$ Fluo3-AM, an acetoxymethyl ester form of the fluorescent dye Fluo-3; Thermo Fisher Scientific Cat F1242) for $30 \mathrm{~min}$ at room temperature and then washed five times. The intracellular $\mathrm{Ca}^{2+}$ transients were evoked by dopamine-hydrochloride (DA, $1 \mathrm{mM}$ final), acetylcholinesterase (ACh, $1 \mathrm{mM}$ final), and glutamate (GLUT, $100 \mu \mathrm{L}$ final). The amplitudes of the $\mathrm{Ca}^{2+}$ related fluorescence transients were expressed relative to the resting fluorescence $(\Delta F / F)$ and were calculated by the formula $\Delta F / F=\left(F_{\text {max }}-F_{\text {rest }}\right) /\left(F_{\text {rest }}-F_{\text {bg }}\right)$ according to Pap et al., 2009 [15]. The Image $J$ program (https://imagej.net/) was used for the calculation of the fluorescence intensities as previously published by Pap et al., 2009 and Mendivil-Perez et al., 2019 [11, 12].

\section{Data Analysis}

Statistical analyses were conducted using the Student-t analysis or one-way ANOVA followed by Bonferroni posthoc comparison calculated with the GraphPad Prism 6 scientific software (GraphPad, Software, Inc. La Jolla, CA, U.S.A.). Statistical significance was accepted at $\star_{p}<0.05 ; * x p<0.01$; $\star * * p<0.001$.

\section{Results}

\section{Characterization of MenSCs}

We first cultured and characterized the morphological, karyotype, immuno-phenotypic features, and differentiation capabilities of MenSCs. Complying with the International Society for Cellular Therapy (ISCT) MSCs criteria [1, 2], MenSCs displayed the typical colony-forming units (Fig. 1A), adherent growth, and fibroblast-like cellular morphology (Fig. 1B). Karyotype analysis showed no chromosomal alterations (Fig. 1C). Flow cytometry analysis showed that MenSCs were positive ( $>95 \%$ of positive cells) for mesenchymal associated markers CD73, CD90, and CD9 (Fig. 1D) but negative ( $<5 \%$ of positive cells) for hematopoietic cell surface antigens CD34/ CD45. Likewise, MenSCs cultured in osteogenic, adipogenic, or chondrogenic induction medium differentiated into osteoblasts (Fig. 1F), adipocytes (Fig. 1H), and chondrocytes (Fig. 1J), respectively, while MenSCs cultured in regular culture medium were undifferentiated (Fig. 1E, $1 \mathrm{G}$ and 1I).

Next, we wanted to evaluate the presence of neural and astrocyte-associated molecules in MenSCs under in minimal culture medium $(\mathrm{MCm})$. Analysis of protein expression by western blotting showed a basal level of NFL (Fig. 2A and 2B), $\beta$-TUB III (Fig. 2A and 2C), GFAP (Fig. 2A and 2D), S100b (Fig. 2A and 2E), ChAT (Fig. 2A and 2F), and TH (Fig. 2A and 2G) proteins in MenSCs culture in MCm at day 0, 4 and 7. Similar observations were found in those cells on day 7 assessed by immunofluorescent microscopy (IFM, Fig. 2H-J).

\section{In vitro transdifferentiation of MenSCs into dopaminergic-like neurons (DALNs) involves the up-regulation of specific neural and dopaminergic proteins.}


To assess the ability of MenSCs to transdifferentiate into DALNs, MSCs were cultured with NeuroForsk medium for 4 or 7 days [14]. Fig. 3 shows a statistically significant increase in the expression level of protein $\beta$-TUB III ( $\sim 0.5-$ and $\sim 0.6-f . i$. , Fig. 3A and 3D), TH ( 1.0- and 1.4-f.i., Fig. 3A and 3F), and DAT ( 2.0- and 1.0-f.i., Fig. $3 \mathrm{~A}$ and $3 \mathrm{G}$ ) but protein expression dramatically decreased in GFAP ( 0.5- and $\sim 0.2$-f.d., Fig. $3 A$ and $3 E$ ) as determined by WB assay and IFM analysis (e.g., $\beta$-TUB III Fig. 3N' and 3T, TH/DAT Fig. 3Q, 3V and 3W). Whereas the expression level of protein NFL (Fig. 3A, 3B, 3l'-K') and MAP2 (Fig. 3A, 3C, 3l"-K") was unaffected by the NeuroForsk medium, the GFAP protein was significantly diminished (Fig. 3A, 3E, $\left.3 L^{\prime}-3 N^{\prime}\right)$. Remarkably, the expression level of protein VChAT increased ( 1.5- and 1.4-f.i., Fig. $3 \mathrm{~A}$ and $3 \mathrm{H}$ ) on days 4 and 7.

To further confirm the dopaminergic lineage of MenSCs- induced DALNs, we analyzed TH and DAT double staining. As shown in Fig. 4, flow cytometry (FC) analysis shows a statistically significant increase in the percentage of TH/ DAT expressing cells at day $4(\sim 14 \%)$ and $7(\sim 26 \%)$ of cell-cultured in NeuroForsk medium compared to MenSCs at day 0 ( $8 \%$, Fig. 4A and 4B). We found no statistical difference $(p<0.05)$ in the level of double expression of TH/ DAT in DALNs at 4 and 7 days of culture.

Dopamine triggers intracellular $\mathrm{Ca}^{2+}$ accumulation in MenSCs-derived DALNs.

Based on the significant increase of dopaminergic cellular populations in MenSCs exposed to NeuroForsk medium, we evaluated the functional response of DALNs under a dopamine stimulus. Therefore, the cytoplasmic $\mathrm{Ca}^{2+}$ accumulation in DALNs was evaluated with Fluo-3-mediated $\mathrm{Ca}^{2+}$ imaging. While DALNs were unaffected by dopamine at day 0 , dopamine-induced a transient intracellular $\mathrm{Ca}^{2+}$ elevation at days 4 and 7 (Fig. 4C and 4D) under the same experimental conditions. Furthermore, the maximal fluorescence change $(\Delta F / F)$ at day 4 was $0.75 \pm 0.05$ folds after $10 \mathrm{sec}$. of dopamine exposure compared to cells at day $0(p<0.05)$, whereas the maximal $\Delta F / F$ at day 7 was $0.25 \pm 0.2$-fold after $20 \mathrm{sec}$. of dopamine addition ( $p<0.05)$ and it remains stable until the 50s (Fig. 4D).

In vitro transdifferentiation of MenSCs into Cholinergic-like neurons (ChLNs) involves the up-regulation of specific neural and cholinergic proteins.

To determine whether MenSCs transdifferentiated into ChLNs, we determined the expression of several molecules associated with neural differentiation in cells treated with Cholinergic-N-Run medium after 0,4 , and 7 days of incubation. Western blot analyzes revealed that the MenSCs at day 0 expressed basal level of protein NFL (Fig. 5A and 5B), MAP2 (Fig. 5A and 5C), $\beta$-TUB III (Fig. 5A and 5D), GFAP (Fig. 5A and 5E), TH (Fig. 5A and 5F), ChAT (Fig. 5A and 5G) and VAChT (Fig. $5 \mathrm{~A}$ and $5 \mathrm{H}$ ). Interestingly, when the cells were exposed to Cholinergic- $\mathrm{N}-\mathrm{Run}$ medium for 4 and 7 days, they significantly increased the levels of NFL ( 1.3- and 
1.4-f.i., Fig. 5B), ChAT ( 1.5- and 1.6-f.i., Fig. 5G) and VAChT ( 2.2- and 2.5-f.i., Fig. $5 \mathrm{H}$ ), while they retained a basal and stable expression of protein MAP2 and $\beta$ TUB III (Fig. 5C and 5D). However, we found the expression level of protein GFAP ( 0.9- and 0.5-f.r., Fig. 5E) and TH ( 0.7- and 0.7-f.r., Fig. 5F) reduced after 4 and 7 days of incubation with the differentiation medium. Similar results were obtained by immunofluorescence analyses (Fig. 5l-5W).

Since the choline acetyltransferase (ChAT) enzyme and vesicular acetylcholine transporter (VAChT) protein are specific cholinergic cell lineage markers [16, 17], we wanted to confirm whether the MenSCs transdifferentiated into ChLNs simultaneously expressed ChAT and VAChT. Accordingly, we establish the percentage of MenSCs derived ChLNs with Cholinergic-N-Run medium and we assessed the proportion (\%) of ChAT/ VAChT immunopositive neurons after the cholinergic induction. Flow cytometry analysis shows a statistically significant increase in the percentage of ChAT/ VAChT expressing cells at day $4(\sim 39 \%)$ and day 7 ( $46 \%)$ of culture exposure compared to MenSCs at day 0 ( $28 \%)$ (Fig. 6A and 6B). We found no statistical difference $(p<0.05)$ in the level of double expression of ChAT/ VAChT in ChLNs at 4 and 7 days of culture.

Acetylcholine triggers intracellular $\mathrm{Ca}^{2+}$ accumulation in MenSCs-derived ChLNs

The above findings encouraged us to evaluate the response of ChLNs cells to acetylcholine culture in Cholinergic-N-Run medium. Therefore, the cytoplasmic $\mathrm{Ca}^{2+}$ accumulation in $\mathrm{ChLNs}$ was evaluated with Fluo-3-mediated $\mathrm{Ca}^{2+}$ imaging. While ChLNs were unaffected by ACh at day 0 of Cholinergic-N-Run medium culture, ACh induced a transient intracellular $\mathrm{Ca}^{2+}$ elevation at day 4 and day 7 (Fig. 6C and 6D) under the same experimental conditions. Furthermore, the maximal fluorescence change (DF/F) at day 4 was $6.14 \pm 1$.5-folds and the maximal DF/F at day 7 was $5.57 \pm 1.2$-fold after $10 \mathrm{sec}$ ACh exposure compared to cells at day 0 ( $p<0.05$, Fig. 6D).

In vitro transdifferentiation of MenSCs into astrocyte-like cells (ALCs) involves the up-regulation of specific glial proteins.

The transdifferentiation of MenSCs into astrocytes was evaluated after 0,4 , and 7 days of incubation with astrocyte medium ${ }^{\circledR}$. As shown in Fig. 7, MenSC-derived ALCs expressed statistically significant increased expression level of S100ß (e.g., 1.2-f.i., Fig. 7A and C) and GFAP ( 0.8- and 0.6-f.i., Fig. 7A and 7D) proteins cultured for 4 and 7 days compared to levels of expression protein of cells cultured at day 0 (Fig. 7C and 7D). Further Western blotting analysis shows that MenSC-derived ALCs expressed almost basal level of protein NFL (Fig. 7A and 7E) and b-TUB III (Fig. 7A and 7F). Similar results were obtained by IMF analyses (Fig. 7G-7P). Since GFAP is the gold standard marker to identify astrocytes and 
S100 $\beta$ indicates a mature stage of astrocytes, we evaluate those markers in the MenSCs-derived ALC population. Accordingly, FC analysis shows a statistically significant increase in GFAP/S100 $\beta$ doublepositive cells in ALCs at day $4(\sim 25 \%)$ and $7(\sim 59 \%)$ of culture compared to cells cultured at day 0 (Fig. $8 \mathrm{~A}$ and $8 \mathrm{~B})$.

Glutamate triggers intracellular $\mathrm{Ca}^{2+}$ accumulation in ALCs

Intracellular astrocyte calcium signaling is triggered by multiple factors, including glutamate at the synaptic cleft, which stimulates $\mathrm{G}$ protein-coupled receptors on the membrane, leading to the production of IP3. IP3 activates IP3R2 receptors on the endoplasmic reticulum (ER), resulting in intracellular calcium release and subsequent exocytosis [18]. Therefore, the cytoplasmic $\mathrm{Ca}^{2+}$ accumulation in ALCs was evaluated with Fluo-3-mediated $\mathrm{Ca}^{2+}$ imaging. While ALCs were unaffected by glutamate at day 0 of Astrocyte medium culture, glutamate-induced a transient intracellular Ca2+ elevation at day 4 and day 7 (Fig. $8 \mathrm{C}$ and $\mathrm{D}$ ) under the same experimental conditions. Furthermore, the maximal fluorescence change $(\Delta F / F)$ at day 4 was $0.10 \pm 0.05$-folds after $120 \mathrm{sec}$. of glutamate exposure compared to cells at day $0(p<0.05)$, whereas the maximal $\Delta F / F$ at day 7 was $0.50 \pm 0.2$-fold after 240 sec. of glutamine addition $(p<0.05)$ and decline slowly.

In vitro trans-differentiation of MenSCs into Neurospheres/spheroids

To determine whether MenSCs transdifferentiate into NSs, we initially determined the morphology and the presence of molecules associated with NSs formation in cells treated with Fast-N-Spheres medium after 0,1 , and 3 days of incubation according to a previous report [11]. As shown in Fig. 9, MenSCs cultured with this specific displayed the typical morphology of NSs as early as 1 day and formed large NSs at 3 days when compared to 0 days of incubation. Interestingly, both the MenSCs ( 0 days) and the formed NSs (at 1 or 3 days) expressed high levels of SOX2 and NESTIN proteins according to western blot analysis (Fig. 9A-C). These results were confirmed by IMF analysis (Fig. 9D-9K).

Next, we wanted to investigate whether large NSs expressed broad ( $\beta$-TUB. III) and specific (TH; ChAT) neural and astrocytic proteins (GFAP). According to western blot analysis, after 3 days of incubation with Fast-N-Spheres medium, NSs significantly increased the expression level of GFAP (Fig. 9L and 9M), TH ( 1.2 f.i., Fig. 9L and 9N), ChAT ( 1.2 f.i., Fig. 9L and 90) and $\beta$-TUB III ( 1.3 f.i., Fig. 9L and 90) protein compared to cells at 0 days of incubation. These results were also confirmed by IMF analysis (Fig. 9Q9X).

\section{Discussion}

The stromal cell-based disease model provides a platform for a better understanding of human neurodegenerative disease mechanisms (e.g., [19]) and the potential discovery of innovative therapeutics (e.g., [20]). As primary human neurons from living subjects are normally not accessible to researchers, there is a pressing need for an alternative source of authentic human neurons which allows modeling of 
neurodegeneration in vitro. Therefore, optimal cell culture conditions and timing are critical for experimental success. Recently, our laboratory has developed three original culture media known as NeuroForsk, Cholinergic-N-Run, and Fast-N-Spheres media to obtain dopaminergic-like (DALNs), cholinergic-like (ChLNs) neurons, and neurospheres (NSs) from UC-WJ-MSCs [11, 12]. By using those culture media, which seems more efficient compared to the already used protocol in developing neurallike cells, we have successfully transdifferentiated MenSCs into functional DALNs, ChLNs, and NSs in 4-7 days of cell culture. Likewise, by using a commercial culture media (e.g., Astrocyte media ${ }^{\circledR}$ ), we also obtained functional astrocyte-like cells (ALCs) in a similar time frame (i.e., 4-7 days). Also, MenSCs can differentiate into various mesoderm cell lineages, such as adipocyte (this work), chondrocytes [21], and osteocytes $[22,23]$ comparable to MSCs obtained from several tissue sources, including perinatal (e.g., WJ-MSCs [24, 25]), bone marrow, adipocyte, dental pulp, and human efflux (e.g., MenSCs [7, 10]), among others. Furthermore, naïve MenSCs (day 0) express basal protein levels of neuronal markers NFL, b-TUB III, TH, DAT, ChAT, VAChT, and MAP2. In contrast to Azedi et al., 2014 [9], we found that MenSCs express GFAP. Moreover, the expression of S100b protein confirms that MenSCs are primed to display glialspecific lineage markers. Differential experimental procedures might explain those divergent results. Taken together, these observations suggest that not only MenSCs differentiate in mesoderm lineages, but also express neuronal precursors typical of ectoderm lineages (i.e., neural lineage). These cellular features together with the fact that MenSCs have no ethical concerns, are easily recovering from women, are unexpensive and time-saving, make MenSCs ideal for disease modeling.

We report for the first time that MenSCs can transdifferentiate into DALNs ( 26\%) cultured in Neuroforsk media for 7 days. Three observations support these findings. First, transdifferentiated DALNs expressed high levels of specific dopaminergic lineage markers $\mathrm{TH}$ - the enzyme necessary for the dopamine production from its precursor L-tyrosine, and DAT - a dopamine transporter plasma membrane glycoprotein, as well as expressed high levels of neuronal-specific protein markers NFL, MAP2, and $\beta$-TUB III. Both DAT and TH expression identifies bona fide dopaminergic neurons [26]. Second, the percentage of protein expression of TH ( 29\% + cells), DAT ( 33\% + cells), and ( 26\% TH/DAT double-positive cells) found in DANLs are comparable or even higher than previously reported from other sources such as those obtained in WJ-MSC cells after 7 days of differentiation with Neuroforsk only ( 25\% TH+ [14]), or bone marrow-MSC ( 26\% TH+; 16\% DAT+), the dental pulp ( 29\% TH+; 23\% DAT+), adipocyte-MSC ( 29\% $\mathrm{TH}+; \sim 23 \% \mathrm{DAT}+)$ after 14 days of differentiation with Forskolin and FGF2 [27]. Finally, dopamine signaling-evoked intracellular $\mathrm{Ca}^{2+}$ concentration changes in DALNs. Interestingly, the expression of TH $(\sim 17 \%)$ and DAT $(\sim 10 \%)$ proteins in naïve MenSCs reveal a strong potential of MSCs to generate DALNs. Moreover, though we found $8 \%$ TH/DAT double-positive cell markers at day 0 , undifferentiated cells do no exhibit a functional phenotype in contrast to DALNs at days 4 and 7 (Fig. 4). This observation can be explained most probably by the absence of dopaminergic $\mathrm{D}_{2}$-receptors in naïve MenSCs. However, mature dopaminergic neurons display an increase of dopaminergic receptors that are linked to synaptogenesis processes and $\mathrm{Ca}^{2+}$ influx [28]. 
Here we also report for the first time that MenSCs transdifferentiate into ChLNs in 4-7 days. Effectively, MenSCs-derived ChLNs significantly expresses cholinergic markers ChAT -the enzyme that catalyzes the biosynthesis of ACh [29], and VAChT - the ACh neurotransmitter transporter [16], two unique markers for cholinergic neurons according to WB, IMF, and FC analysis. In agreement with others, who have demonstrated that MSCs from dental pulp [30], adipose tissue [31], and UC-WJ [12] can transdifferentiate into ChNs/ ChLNs, we show that MenSCs readily transdifferentiated into functional ChLNs. Similar to UCWJ-MSCs-derived ChLNs [12], the MenSCs-derived ChLNs are responsive to ACh stimuli on days 4 and 7. Interestingly, though naïve MenSCs express both cholinergic ChAT/ VAChT and ACh receptors [32], they are unresponsive to ACh at day 0 . These observations suggest that the presence/expression of cholinergic markers in undifferentiated MenSCs (i.e., ChAT/ VAChT/ AChRs) are not yet functional indicating a cellular immature state that necessitates an adequate micro-environmental stimulus, as provided by Cholinergic-N-Run media, to express functional (responsive) AChRs [33]. Interestingly, ChLNs maintained the expression of general neural lineage markers (e.g., NFL, b-TUB III) whereas other specific cell lineages markers diminished (e.g., GFAP, TH) at day 7 of transdifferentiating. Therefore, we conclude that MenSCs-derived ChLNs are mature neural cells obtained timelessly.

In contrast to Mendivil-Perez and co-workers [12], who reported that MSCs from WJ-MSCs generated $\sim 76 \%$ ChLNs according to double ChAT /VAChT analysis, we found that under similar experimental conditions MenSCs derived from MenB produced $\sim \mathbf{4 6} \%$ ChLNs, a much less percentage yield when compared to WJ-MSCs-derived ChLNs (i.e., $-40 \%$ reduction). These observations suggest that MSCs from UC are more efficient to produce ChLNs than MenSCs derived from MenB. However, MenSCs-derived ChLNs responded faster to ACh stimulus (maximal (DF/F) cytoplasmic $\left(\mathrm{Ca}^{2+}\right)_{\mathrm{i}}$ elevation at day 7 after 10s) than WJ-MSCs-derived ChLNs (maximal (DF/F) cytoplasmic $\left(\mathrm{Ca}^{2+}\right)_{\mathrm{i}}$ elevation at day 7 after 40s). Despite these, both derived ChLNs responded almost $100 \%$ to ACh. Therefore, MenSCs are suitable for pharmacological studies.

Astrocytes are specialized glial cells of the central nervous system that play essential roles in the maintenance, metabolism, and immune response and assistance of neuronal cells in the brain [34-36]. Therefore, astrocytes are deeply implicated in the neuropathology of neurodegenerative disorders [37]. We confirm that MenSCs transdifferentiate into glial cells [9]. Specifically, we report for the first time that MenSCs-derived astrocyte-like cells (ALCs) are obtained in 7 days of culture in Astrocyte medium ${ }^{\circledR}$. As expected, ALCs expressed high expression levels of protein GFAP - uniquely found in astrocytes [38] and S100b [39] according to WB, FC, and IMF assays. Interestingly, the expression of b-TUB and NFL in ALCs were not affected by cultural conditions. Given that b-TUB is constitutively co-expressed with GFAP in midgestational human fetal astrocytes [40] and astrocyte in culture [41], it is not surprising that we detect b-TUB and NFL -two specific neuronal markers in ALCs. These observations suggest that the expression of b-TUB, NFL, and NESTIN [40] in ALCs is a common feature with neuronal cells.

Although the commercial Astrocyte medium ${ }^{\circledR}$ is regularly used to support the growth and maintenance of primary human astrocytes

(https://www.thermofisher.com/order/catalog/product/A1261301\#/A1261301), we found that it can be

Page $11 / 26$ 
used for the transdifferentiation of MenSCs into ALCs in 7 days of culture $\left(59 \% \mathrm{GFAP}^{+} / \mathrm{S} 100 \mathrm{~b}^{+}\right)$. This is a fast, direct, economical, and time-saving protocol for the obtention of ALCs. Furthermore, ALCs tested at days 4 and 7 but not at day 0 were responsive to GLUT stimulus (Fig.8). We conclude that ALCs represent functional glial cells.

Neurospheres (NSs) are 3D structures composed of free-floating conglomerates of neural progenitor cells (NPCs) derived from either isolated primary tissue (e.g., embryonic tissue) or from induced pluripotent stem cells (iPSCs) and grown for limited periods. Therefore, NSs provide an unmatched platform to evaluate the stem-/stromal-cell-like behavior of neurogenic tissue and can be used in a variety of in vitro experiments to delineate the molecular and cellular characteristics of neuronal cells for in vivo transplantation in neurodegenerative disorders (e.g., [42]). We have reported a fast and easy method to obtain NSs from UC-MSCs in 24 hr by using the Fast-N-Spheres medium [11]. Under similar experimental conditions, we have been able to transdifferentiate MenSCs into NSs phenotypically similar to MSCsderived NSs. However, while the MenSCs-derived NSs were NESTIN and SOX-2 positive -a key transcription factor in the regulation of pluripotency and neural differentiation [43], the MSCs-derived NSs were NESTIN positive but SOX-2 negative [11]. These observations suggest that NSs derived from MenB conserved those specific markers similar to those observed in NSs from neurogenic niches in cerebral tissue [44]. Therefore, MenSCs-derived NSs are a more reliable 3D cellular source for neurodevelopmental studies. Moreover, in agreement with previous data [8], the MenSCs-derived NSs cultured for 3 days significantly increased the expression of neuronal (e.g., b-TUB III) as well astrocytic (e.g., GFAP) markers. We report for the first time that MenSCs-derived NSs also express the specific neuronal lineage dopaminergic marker TH and cholinergic marker ChAT. Taken together these results suggest that MenSCs-derived NSs can highly regulate tri-neuronal lineage proteins typical of the dopaminergic, cholinergic, and glial neuronal cells, which might represent an excellent strategy not only to studying the tissular pathology of AD and PD but also to be used as starting material for the development of more complex structures such as organoids [45]. Therefore, as depicted in Fig. 10, MenSCs are a unique and reliable biological source for derivation of the most important neural cells such as dopaminergic, cholinergic, and astrocytic neuronal cells implicated in neurodegeneration (e.g., Alzheimer's and Parkinson's diseases).

\section{Declarations}

\section{Funding}

DQ-E and VS-M are doctoral students and CQ-Q is a master's student from the Neuroscience Study Program at the Basic Biomedical Sciences Academic Corporation, UdeA. MM-P is an associated researcher (contract \#749-2018). DQ-E is founded by the Committee for Development and Research CODI-UdeA (grants \#2017-15829). VS-M is funded by the 2019 Bicentennial Doctoral Excellence 
Scholarship, MinCiencias-Colombia. CQ-Q is funded by the Young Research Award Program from MinCiencia (grants \# 111580762912).

\section{Conflicts of Interest}

The authors have no conflicts of interest to declare that are relevant to the content of this article.

\section{Author Contributions}

DQ-E, VS-M, CQ-Q, and MM-P performed MenSCs-derived dopaminergic, cholinergic, astrocytic, and neurospheres in vitro experiments, respectively. All performed in vitro data analysis and wrote the first draft. MJdelR/ CV-P conceived the in vitro experiments, contributed with reagents, data analysis, wrote, reviewed, and edited the paper. All authors read and approved the final version of the paper.

\section{Ethics approval}

Menstrual specimen donors provided a signed informed consent approved by the Ethics Committee of the Sede de Investigación Universitaria -SIU-, University of Antioquia, Medellín, Colombia (act 202010854).

\section{Availability of data and material}

All datasets generated for this study are included in the manuscript and/or the supplementary files.

\section{Consent to participate}

Not applicable

\section{Consent for Publication}

Not applicable

\section{Acknowledgments}

This work was supported by MinCiencias (grants \#1115-807-62912, contract \#749-2018).

\section{References}

1. Dominici, M., et al., Minimal criteria for defining multipotent mesenchymal stromal cells. The International Society for Cellular Therapy position statement. Cytotherapy, 2006. 8(4): p. 315-7.

2. Viswanathan, S., et al., Mesenchymal stem versus stromal cells: International Society for Cell \& Gene Therapy (ISCT $\AA)$ Mesenchymal Stromal Cell committee position statement on nomenclature. Cytotherapy, 2019. 21(10): p. 1019-1024. 
3. Staff, N.P., D.T. Jones, and W. Singer, Mesenchymal Stromal Cell Therapies for Neurodegenerative Diseases. Mayo Clin Proc, 2019. 94(5): p. 892-905.

4. Choudhary, P., A. Gupta, and S. Singh, Therapeutic Advancement in Neuronal Transdifferentiation of Mesenchymal Stromal Cells for Neurological Disorders. J Mol Neurosci, 2020.

5. Shariati, A., et al., Mesenchymal stromal cells (MSCs) for neurodegenerative disease: A promising frontier. Eur J Cell Biol, 2020. 99(6): p. 151097.

6. Zhao, Y., et al., Transplantation of Human Menstrual Blood-Derived Mesenchymal Stem Cells Alleviates Alzheimer's Disease-Like Pathology in APP/PS1 Transgenic Mice. Front Mol Neurosci, 2018. 11: p. 140.

7. Chen, L., et al., Menstrual blood-derived stem cells: toward therapeutic mechanisms, novel strategies, and future perspectives in the treatment of diseases. Stem Cell Res Ther, 2019. 10(1): p. 406.

8. Azedi, F., et al., Comparative capability of menstrual blood versus bone marrow derived stem cells in neural differentiation. Mol Biol Rep, 2017. 44(1): p. 169-182.

9. Azedi, F., et al., Differentiation potential of menstrual blood-versus bone marrow-stem cells into gliallike cells. Cell Biol Int, 2014. 38(5): p. 615-24.

10. Bozorgmehr, M., et al., Endometrial and Menstrual Blood Mesenchymal Stem/Stromal Cells: Biological Properties and Clinical Application. Front Cell Dev Biol, 2020. 8: p. 497.

11. Bonilla-Porras, A.R., C. Velez-Pardo, and M. Jimenez-Del-Rio, Fast transdifferentiation of human Wharton's jelly mesenchymal stem cells into neurospheres and nerve-like cells. J Neurosci Methods, 2017. 282: p. 52-60.

12. Mendivil-Perez, M., C. Velez-Pardo, and M. Jimenez-Del-Rio, Direct transdifferentiation of human Wharton's jelly mesenchymal stromal cells into cholinergic-like neurons. J Neurosci Methods, 2019. 312: p. 126-138.

13. Liu, Y., et al., Biological characteristics of human menstrual blood-derived endometrial stem cells. J Cell Mol Med, 2018. 22(3): p. 1627-1639.

14. Mendivil-Perez, M., C. Velez-Pardo, and M. Jimenez-Del-Rio, Neuroprotective Effect of the LRRK2 Kinase Inhibitor PF-06447475 in Human Nerve-Like Differentiated Cells Exposed to Oxidative Stress Stimuli: Implications for Parkinson's Disease. Neurochem Res, 2016. 41(10): p. 2675-2692.

15. Pap, P., et al., Cytoplasmic $\mathrm{Ca}(2+)$ concentration changes evoked by cholinergic stimulation in primary astrocyte cultures prepared from the rat cochlear nucleus. Hear Res, 2009. 255(1-2): p. 73-83.

16. Arvidsson, U., et al., Vesicular acetylcholine transporter (VAChT) protein: a novel and unique marker for cholinergic neurons in the central and peripheral nervous systems. J Comp Neurol, 1997. 378(4): p. 454-67.

17. Oda, Y., Choline acety/transferase: the structure, distribution and pathologic changes in the central nervous system. Pathol Int, 1999. 49(11): p. 921-37.

18. Jones, J.R., et al., Mutations in GFAP Disrupt the Distribution and Function of Organelles in Human Astrocytes. Cell Rep, 2018. 25(4): p. 947-958.e4. 
19. Soto-Mercado, V., et al., Cholinergic-like neurons carrying PSEN1 E280A mutation from familial Alzheimer's disease reveal intraneuronal SAPP $\beta$ fragments accumulation, hyperphosphorylation of TAU, oxidative stress, apoptosis and Ca2+dysregulation: Therapeutic implications. PLoS One, 2020. 15(5): p. e0221669.

20. Soto-Mercado, V., et al., Multi-Target Effects of the Cannabinoid CP55940 on Familial Alzheimer's Disease PSEN1 E280A Cholinergic-Like Neurons: Role of CB1 Receptor. J Alzheimers Dis, 2020.

21. Kazemnejad, S., et al., Characterization and chondrogenic differentiation of menstrual blood-derived stem cells on a nanofibrous scaffold. Int J Artif Organs, 2012. 35(1): p. 55-66.

22. Darzi, S., et al., Osteogenic differentiation of stem cells derived from menstrual blood versus bone marrow in the presence of human platelet releasate. Tissue Eng Part A, 2012. 18(15-16): p. 1720-8.

23. Karadas, O., et al., Collagen scaffolds with in situ-grown calcium phosphate for osteogenic differentiation of Wharton's jelly and menstrual blood stem cells. J Tissue Eng Regen Med, 2014. 8(7): p. 534-45.

24. Troyer, D.L. and M.L. Weiss, Wharton's jelly-derived cells are a primitive stromal cell population. Stem Cells, 2008. 26(3): p. 591-9.

25. Davies, J.E., J.T. Walker, and A. Keating, Concise Review: Wharton's Jelly: The Rich, but Enigmatic, Source of Mesenchymal Stromal Cells. Stem Cells Transl Med, 2017. 6(7): p. 1620-1630.

26. Lammel, S., et al., Diversity of transgenic mouse models for selective targeting of midbrain dopamine neurons. Neuron, 2015. 85(2): p. 429-38.

27. Singh, M., et al., Evaluation of Priming Efficiency of Forskolin in Tissue-Specific Human Mesenchymal Stem Cells into Dopaminergic Neurons: An In Vitro Comparative Study. Cells, 2020. $9(9)$.

28. Catoni, C., T. Calì, and M. Brini, Calcium, Dopamine and Neuronal Calcium Sensor 1: Their Contribution to Parkinson's Disease. Front Mol Neurosci, 2019. 12: p. 55.

29. Deutch, A. and R. Roth, Chapter 7 - Pharmacology and Biochemistry of Synaptic Transmission: Classical Transmitters, in From Molecules to Networks (Third Edition), R.H. John H. Byrne, M. Neal Waxham, Editor. 2014. p. 207-237.

30. Kang, Y.H., et al., Comparative analysis of three different protocols for cholinergic neuron differentiation in vitro using mesenchymal stem cells from human dental pulp. Anim Cells Syst (Seoul), 2019. 23(4): p. 275-287.

31. Marei, H.E.S., et al., Cholinergic and dopaminergic neuronal differentiation of human adipose tissue derived mesenchymal stem cells. J Cell Physiol, 2018. 233(2): p. 936-945.

32. Hoogduijn, M.J., A. Cheng, and P.G. Genever, Functional nicotinic and muscarinic receptors on mesenchymal stem cells. Stem Cells Dev, 2009. 18(1): p. 103-12.

33. Castell, X., et al., Exploring the regulation of the expression of ChAT and VAChT genes in NG108-15 cells: implication of PKA and PI3K signaling pathways. Neurochem Res, 2003. 28(3-4): p. 557-64. 
34. Vasile, F., E. Dossi, and N. Rouach, Human astrocytes: structure and functions in the healthy brain. Brain Struct Funct, 2017. 222(5): p. 2017-2029.

35. Liddelow, S.A. and B.A. Barres, Reactive Astrocytes: Production, Function, and Therapeutic Potential. Immunity, 2017. 46(6): p. 957-967.

36. Dienel, G.A. and D.L. Rothman, Reevaluation of Astrocyte-Neuron Energy Metabolism with Astrocyte Volume Fraction Correction: Impact on Cellular Glucose Oxidation Rates, Glutamate-Glutamine Cycle Energetics, Glycogen Levels and Utilization Rates vs. Exercising Muscle, and Na. Neurochem Res, 2020. 45(11): p. 2607-2630.

37. Acioglu, C., L. Li, and S. Elkabes, Contribution of astrocytes to neuropathology of neurodegenerative diseases. Brain Res, 2021: p. 147291.

38. Yang, Z. and K.K. Wang, Glial fibrillary acidic protein: from intermediate filament assembly and gliosis to neurobiomarker. Trends Neurosci, 2015. 38(6): p. 364-74.

39. Michetti, F., et al., The S100B story: from biomarker to active factor in neural injury. J Neurochem, 2019. 148(2): p. 168-187.

40. Dráberová, E., et al., Class III beta-tubulin is constitutively coexpressed with glial fibrillary acidic protein and nestin in midgestational human fetal astrocytes: implications for phenotypic identity. $\mathrm{J}$ Neuropathol Exp Neurol, 2008. 67(4): p. 341-54.

41. Knight, V.B. and E.E. Serrano, Post-Translational Tubulin Modifications in Human Astrocyte Cultures. Neurochem Res, 2017. 42(9): p. 2566-2576.

42. Wang, S.S., J. Jia, and Z. Wang, Mesenchymal Stem Cell-Derived Extracellular Vesicles Suppresses iNOS Expression and Ameliorates Neural Impairment in Alzheimer's Disease Mice. J Alzheimers Dis, 2018. 61(3): p. 1005-1013.

43. Zhang, S. and W. Cui, Sox2, a key factor in the regulation of pluripotency and neural differentiation. World J Stem Cells, 2014. 6(3): p. 305-11.

44. Soares, R., et al., Isolation and Expansion of Neurospheres from Postnatal (P1-3) Mouse Neurogenic Niches. J Vis Exp, 2020(159).

45. Watanabe, F., et al., Generation of Neurosphere-Derived Organoid-Like-Aggregates (NEDAS) from Neural Stem Cells. Curr Protoc, 2021. 1(2): p. e15.

\section{Figures}



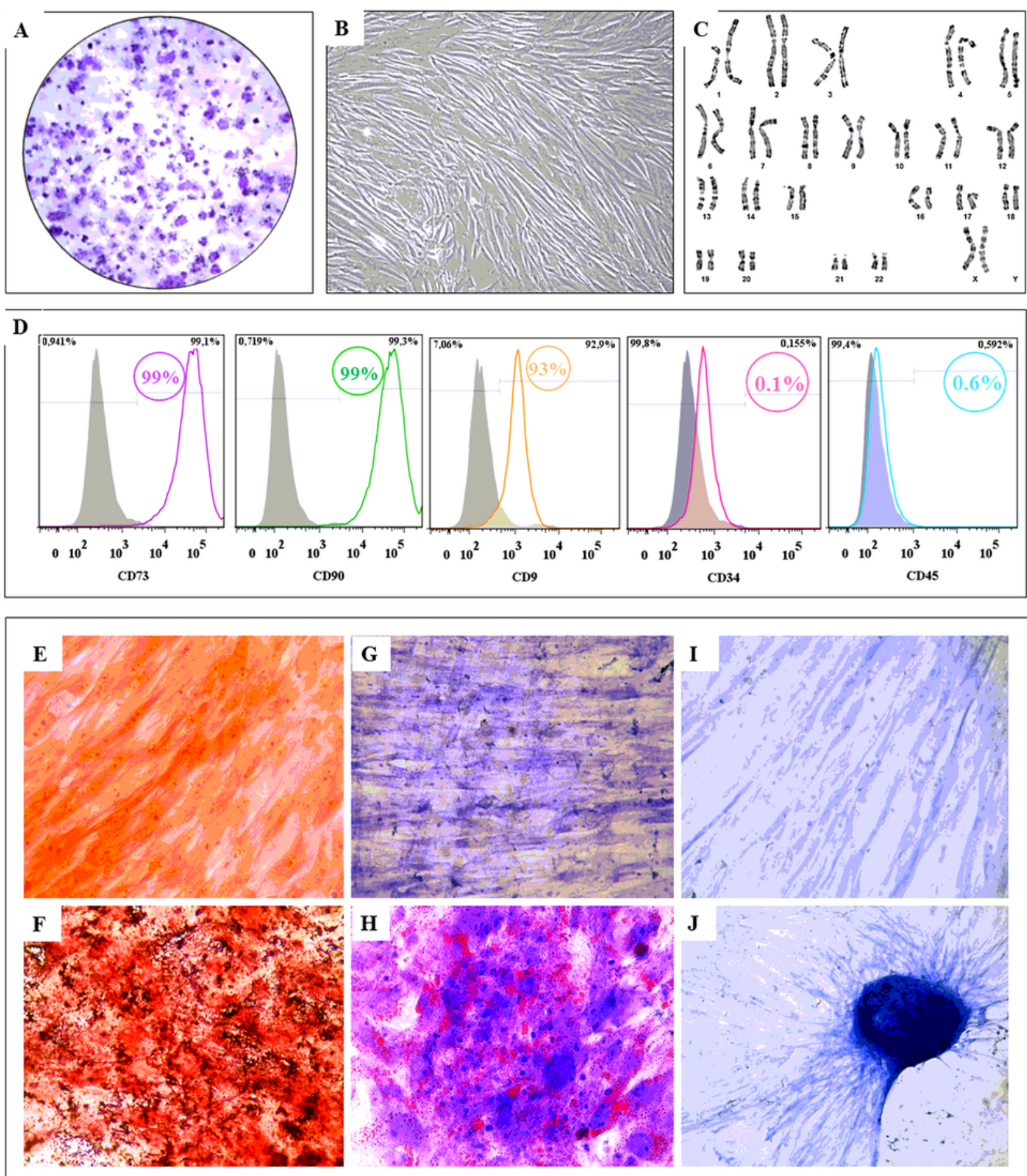

Figure 1

\section{Figure 1}

Characterization of MenSCs. (A) Representative images showing the colony-forming units; (B) adherent growth and fibroblast-like morphology typical of MenSCs. (C) Karyotype analysis performed at passage 2 showing chromosomal normality (46XX). (D) Flow cytometry analyses showing the percentage of doublepositive CD9/ CD73/ CD90/ CD34 and CD45 MenSCs. (E) Von Kossa negatively stained undifferentiated MenSCs grown on a regular culture medium. (F) Von Kossa positively stained osteoblasts differentiated 
from MenSCs showing silver intracellular precipitates. (G) Oil-Red-O negatively stained undifferentiated MenSCs grown on regular culture medium. $(\mathrm{H})$ Oil-Red-O positively stained adipocytes differentiated from MenSCs showing intracellular red lipidic vacuoles. (I) Toluidine blue negatively stained undifferentiated MenSCs grown on regular culture medium. $(\mathrm{J})$ Toluidine blue positively stained chondrocytes differentiated from MenSCs showing extracellular glycoprotein matrix. Image magnification, 400x. The images represent 1 out of 3 independent experiments.

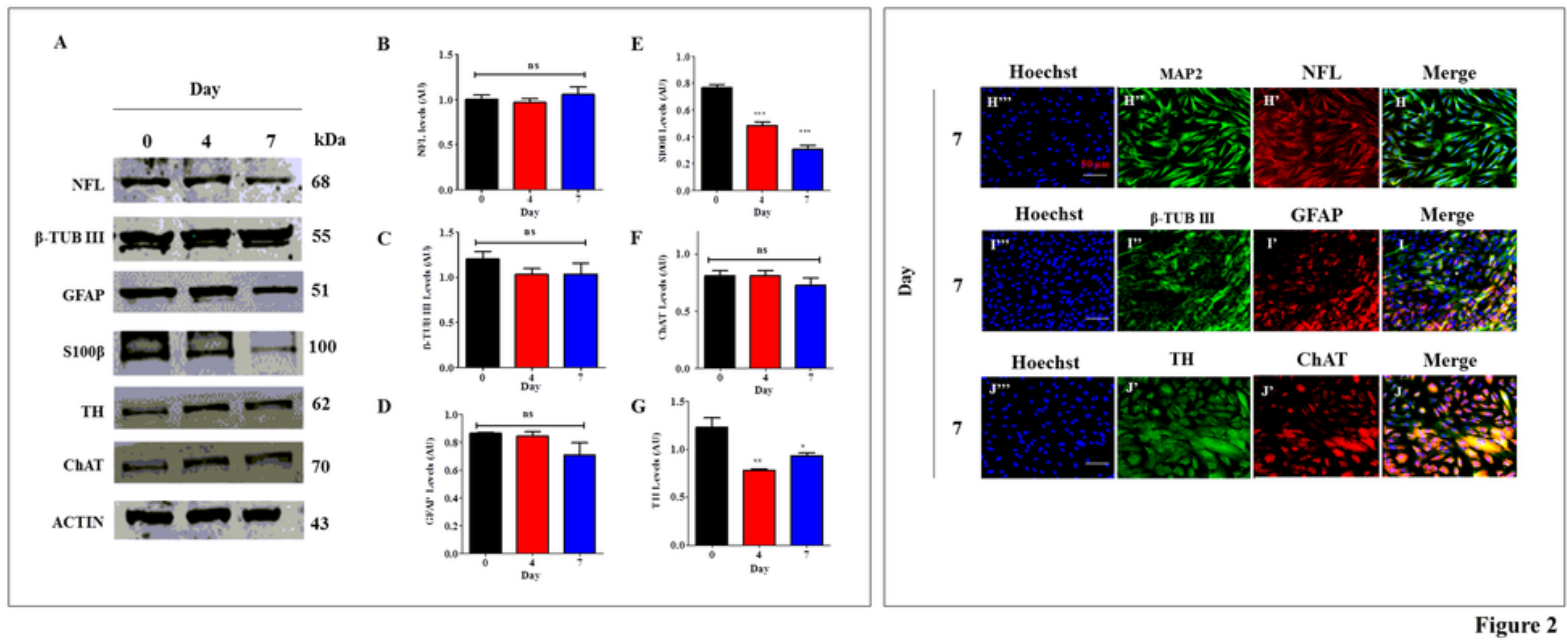

\section{Figure 2}

Western blot and immunofluorescence analysis of undifferentiated MenSCs MenSCs were cultured in $\mathrm{MCm}$ as described in the Materials and Methods section for 0,4 , and 7 days. After this time, the proteins in the extracts were blotted with primary antibodies against NFL, $\beta$-TUB III, GFAP, S100 $\beta, T H$, ChAT, and actin proteins. The intensities of the western blot bands shown in (A) were measured (B, $C, D, E, F$, and $G$ ) by an infrared imaging system (Odyssey, LI-COR), and the intensity was normalized to that of actin. (H-J) At day 0 , cells were double-stained as indicated in the figure with primary antibodies against MAP2 (green; H") and NFL (red; H'), $\beta$-TUB III (green; I") and GFAP (red; I') or TH (green; J") and ChAT (red; J'). The nuclei were stained with Hoechst 33342 (blue; $\mathrm{H}^{\prime \prime}-\mathrm{J}$ '”). (R-W). Data are expressed as the mean \pm SD; ${ }^{*} p<0.05 ;{ }^{* *} p<0.01 ;{ }^{* \star *} p<0.001$. The blots and figures represent 1 out of 3 independent experiments. Image magnification, 200x. 

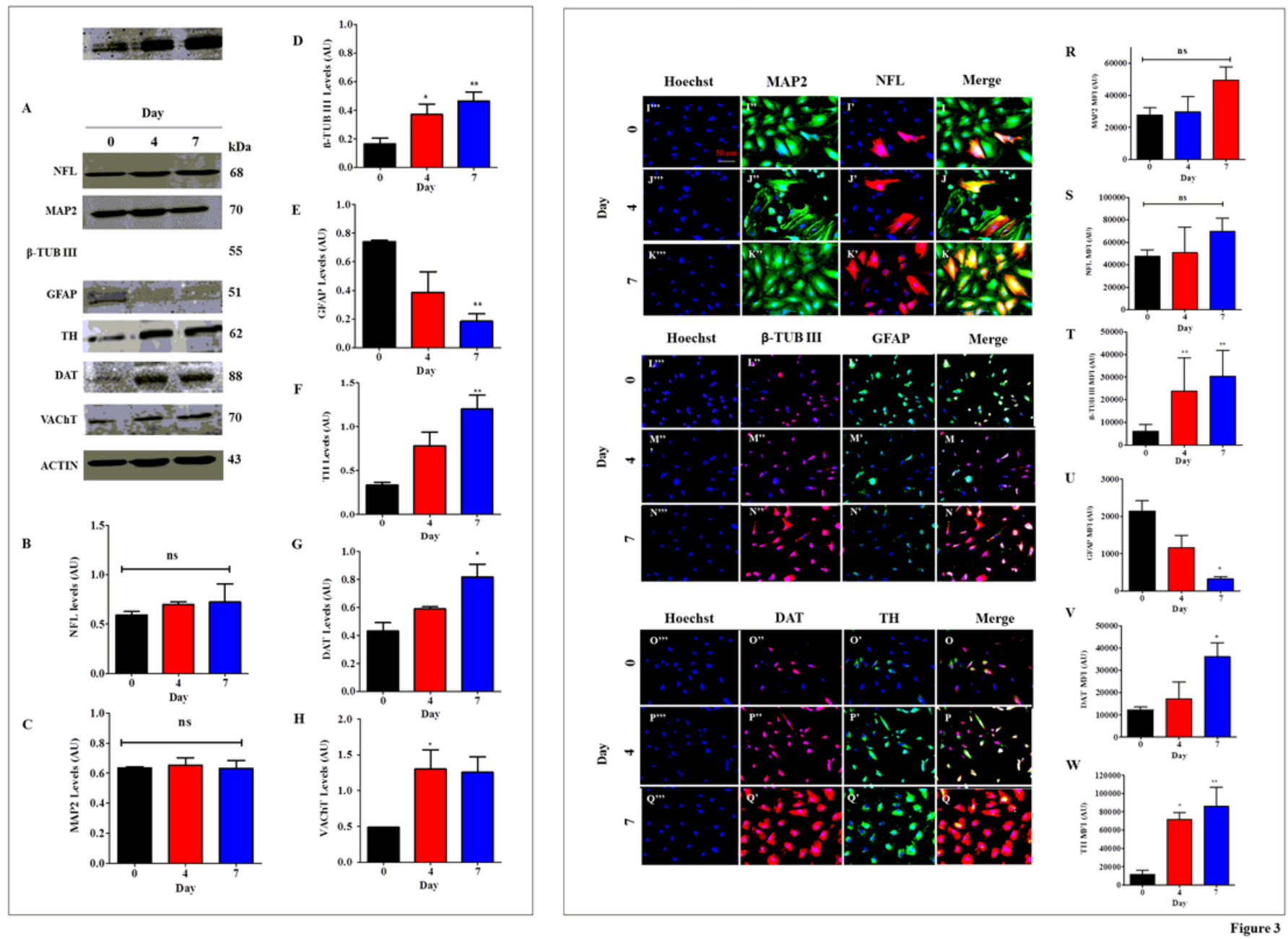

\section{Figure 3}

Western blot and immunofluorescence analysis of Dopaminergic-like differentiation of MenSCs MenSCs were cultured in NeuroForsk medium as described in the Materials and Methods section for 0,4 , and 7 days. After this time, the proteins in the extracts were blotted with primary antibodies against NFL, MAP2, $\beta$-TUB III, GFAP, TH, DAT, VAChT, and actin proteins. The intensities of the western blot bands shown in (A) were measured (B, C, D, E, F, G, and $H$ ) by an infrared imaging system (Odyssey, LI-COR), and the intensity was normalized to that of actin. (I-Q) Cells were double-stained as indicated in the figure with primary antibodies against MAP2 (green; I"-K") and NFL (red; I'-K'), $\beta$-TUB III (green; L"-N") and GFAP (red; L'-N') or DAT (red; O"-Q") and TH (green; O'-Q'). The nuclei were stained with Hoechst 33342 (blue; l'"-Q"'). (R-W) Mean Fluorescence Intensity (MFI) quantification of images obtained by immunofluorescence analysis. Data are expressed as the mean $\pm S D ;{ }^{*} p<0.05 ;{ }^{* \star} p<0.01 ;{ }^{* \star *} p<0.001$. The blots and figures represent 1 out of 3 independent experiments. Image magnification, 200x. 

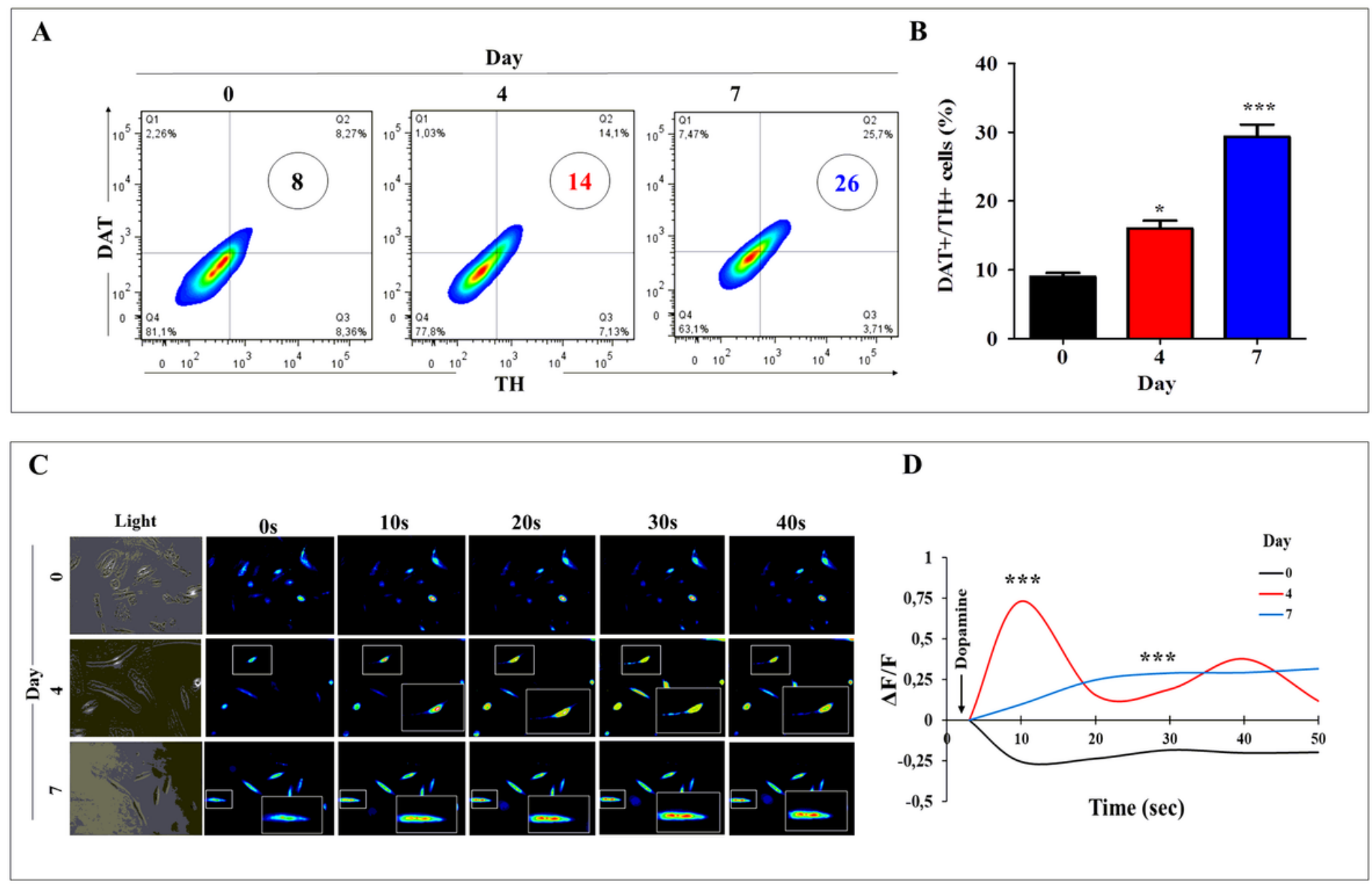

Figure 4

\section{Figure 4}

TH and DAT percentage expression and dopamine (DA) upregulation of cytoplasmic Ca2+ concentration in MenSCs differentiated into Dopaminergic-like cells. MenSCs were cultured in NeuroForsk medium as described in the Materials and Methods section for 0, 4, and 7 days. Representative density plot figures showing the DAT/TH double-positive population of NeuroForsk medium cultured MenSCs at day 0, 4, and 7 (A). (B) Percentage of DAT/TH double-positive cells. (C) Time-lapse images (0, 10, 20, 30 and $40 \mathrm{~s})$ of Ca2+ fluorescence in differentiating MenSC at days 0, 4 and 7 ( $\mathrm{n}=30$ cells imaged, $\mathrm{N}=3$ dishes) as a response to DA treatment. DA was puffed into the culture at $0 \mathrm{~s}$ (arrow). Then, the Ca2+ fluorescence of cells was monitored at indicated times. Color contrast indicates fluorescence intensity: dark blue < light blue < green < yellow < red. (D) Normalized mean fluorescence signal $(\Delta F / F)$ over time, indicating temporal cytoplasmic Ca2+ elevation in response to DA treatment. Data are presented as means \pm SD. ${ }^{*} p<0.05 ;{ }^{* *} p<0.01$. Image magnification 200x. 

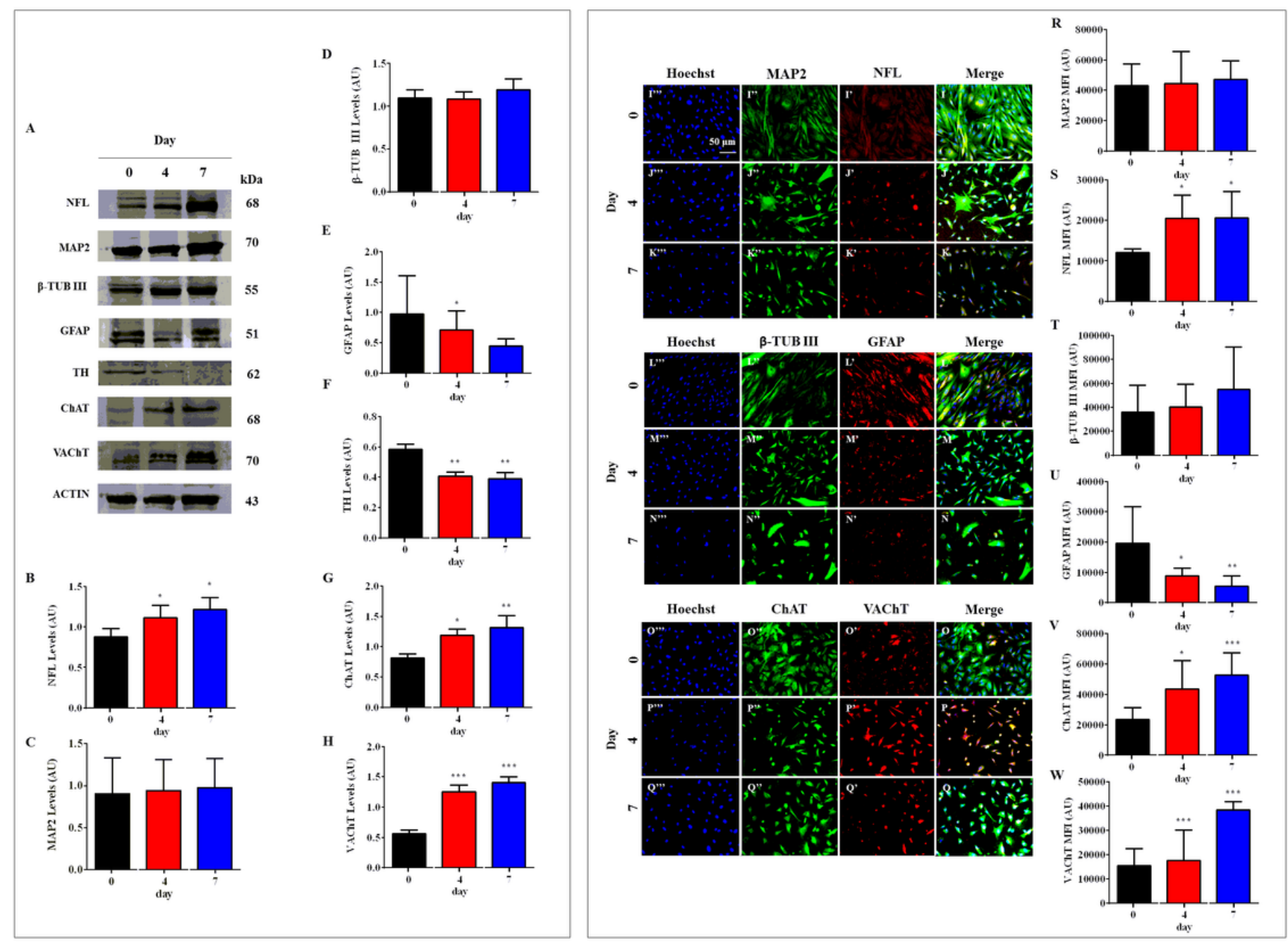

Figure 5

\section{Figure 5}

Western blot and immunofluorescence analysis of Cholinergic-like differentiation of MenSCs MenSCs were cultured in Ch-N-Rm as described in the Materials and Methods section for 0,4 , and 7 days. After this time, the proteins in the extracts were blotted with primary antibodies against NFL, MAP2, $\beta$-TUB III, GFAP, TH, ChAT, VAChT, and actin proteins. The intensities of the western blot bands shown in (A) were measured (B, C, D, E, F, G, and H) by an infrared imaging system (Odyssey, LI-COR), and the intensity was normalized to that of actin. (I-Q) Cells were double-stained as indicated in the figure with primary antibodies against MAP2 (green; I"-K") and NFL (red; I'-K'), $\beta$-TUB III (green; L"-N") and GFAP (red; L'-N') or ChAT (green; O"-Q") and VAChT (red; O'-Q'). The nuclei were stained with Hoechst 33342 (blue; I'--Q'"). (RW) Mean Fluorescence Intensity (MFI) quantification of images obtained by immunofluorescence analysis. Data are expressed as the mean $\pm S D ;{ }^{*} p<0.05 ;{ }^{* *} p<0.01 ; * \star * p<0.001$. The blots and figures represent 1 out of 3 independent experiments. Image magnification, 200x. 

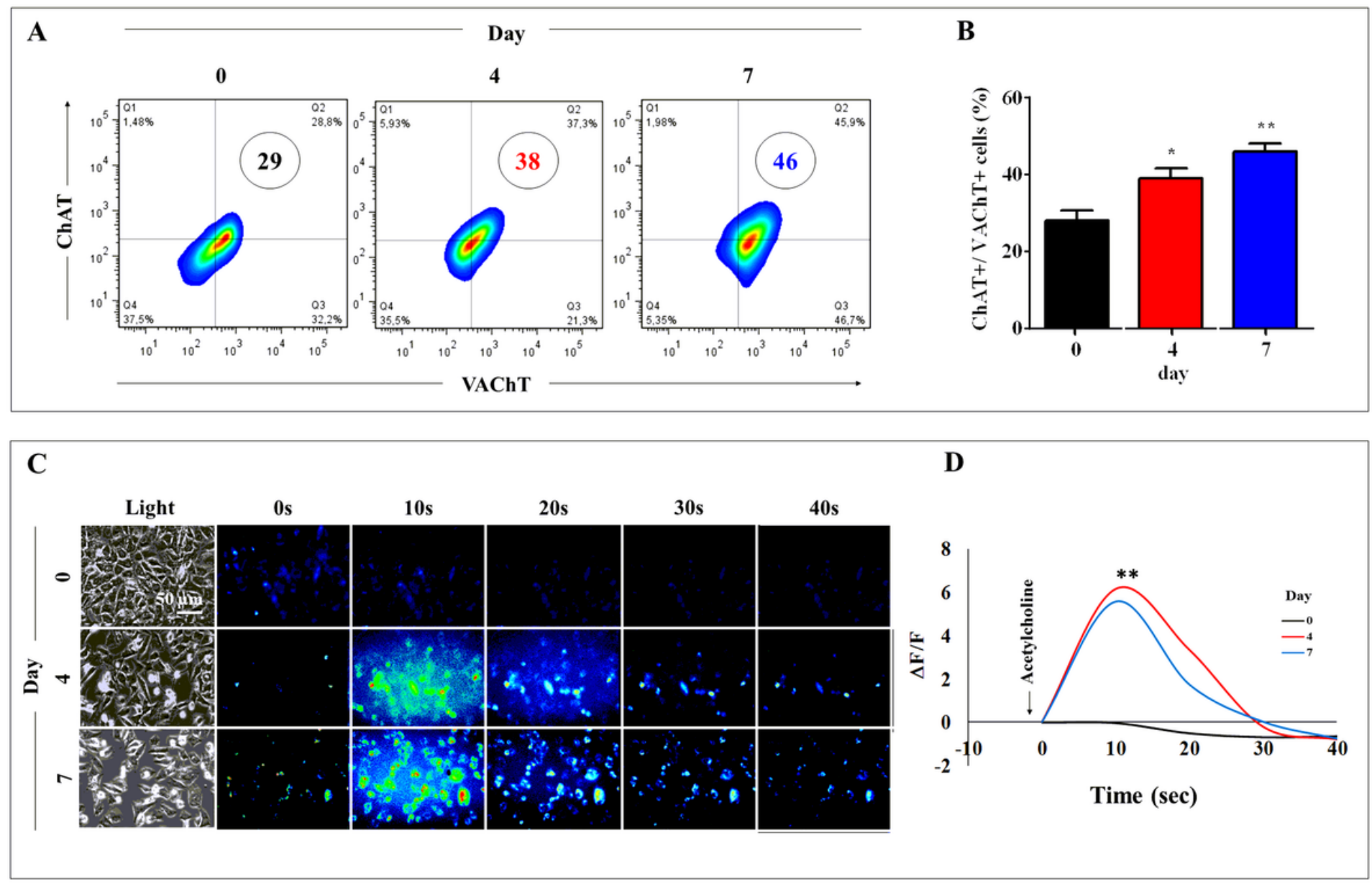

Figure 6

\section{Figure 6}

ChAT and VAChT percentage expression and acetylcholine (ACh) upregulation of cytoplasmic Ca2+ concentration in MenSCs differentiated into Cholinergic-like cells. MenSCs were cultured in Ch-N-Rm as described in the Materials and Methods section for 0, 4, and 7 days. Representative density plot figures showing the ChAT/ VAChT double-positive population of Ch-N-Rm cultured MenSCs at day 0, 4, and 7 (A).

(B) Percentage of ChAT/ VAChT double-positive cells. (C) Time-lapse images (0, 10, 20, 30 and $40 \mathrm{~s})$ of Ca2+ fluorescence in differentiating MenSC at days 0, 4 and 7 ( $n=30$ cells imaged, $N=3$ dishes) as a response to ACh treatment. ACh was puffed into the culture at $0 \mathrm{~s}$ (arrow). Then, the Ca2+ fluorescence of cells was monitored at indicated times. Color contrast indicates fluorescence intensity: dark blue < light blue < green < yellow < red. (D) Normalized mean fluorescence signal $(\Delta F / F)$ over time, indicating temporal cytoplasmic Ca2+ elevation in response to ACh treatment. Data are presented as means \pm SD. ${ }^{*} p<0.05 ;{ }^{* *} p<0.01$. Image magnification 200x. 

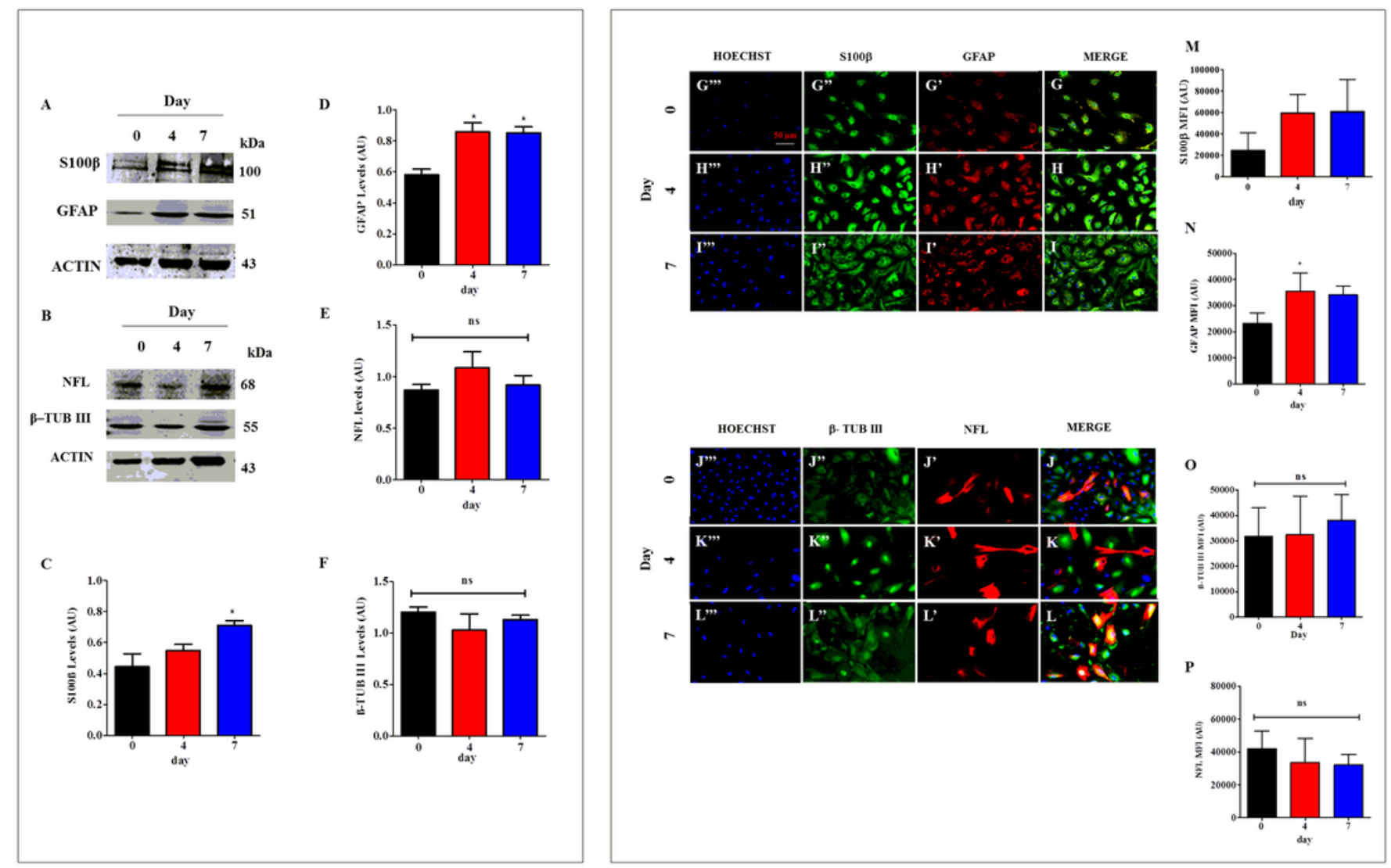

Figure 7

\section{Figure 7}

Western blot and immunofluorescence analysis of Astrocyte-like cells differentiation of MenSCs MenSCs were cultured in Astrocyte medium as described in the Materials and Methods section for 0,4 , and 7 days. After this time, the proteins in the extracts were blotted with primary antibodies against GFAP, S100 $\beta, N F L, \beta$-TUB III, GFAP, and actin proteins. The intensities of the western blot bands shown in (A, B) were measured (C, D, E, and F) by an infrared imaging system (Odyssey, LI-COR), and the intensity was normalized to that of actin. (G-L) Cells were double-stained as indicated in the figure with primary antibodies against S100 $\beta$ (green; G"-I") and GFAP (red; G'-I'), $\beta$-TUB III (green; J"-L") and NFL (red; J'-L'). The nuclei were stained with Hoechst 33342 (blue; G"'-L'"). (M-P) Mean Fluorescence Intensity (MFI) quantification of images obtained by immunofluorescence analysis. Data are expressed as the mean \pm $S D ;{ }^{*}<0.05 ;{ }^{*} p<0.01 ;{ }^{* \star} p<0.001$. The blots and figures represent 1 out of 3 independent experiments. Image magnification, 200x. 

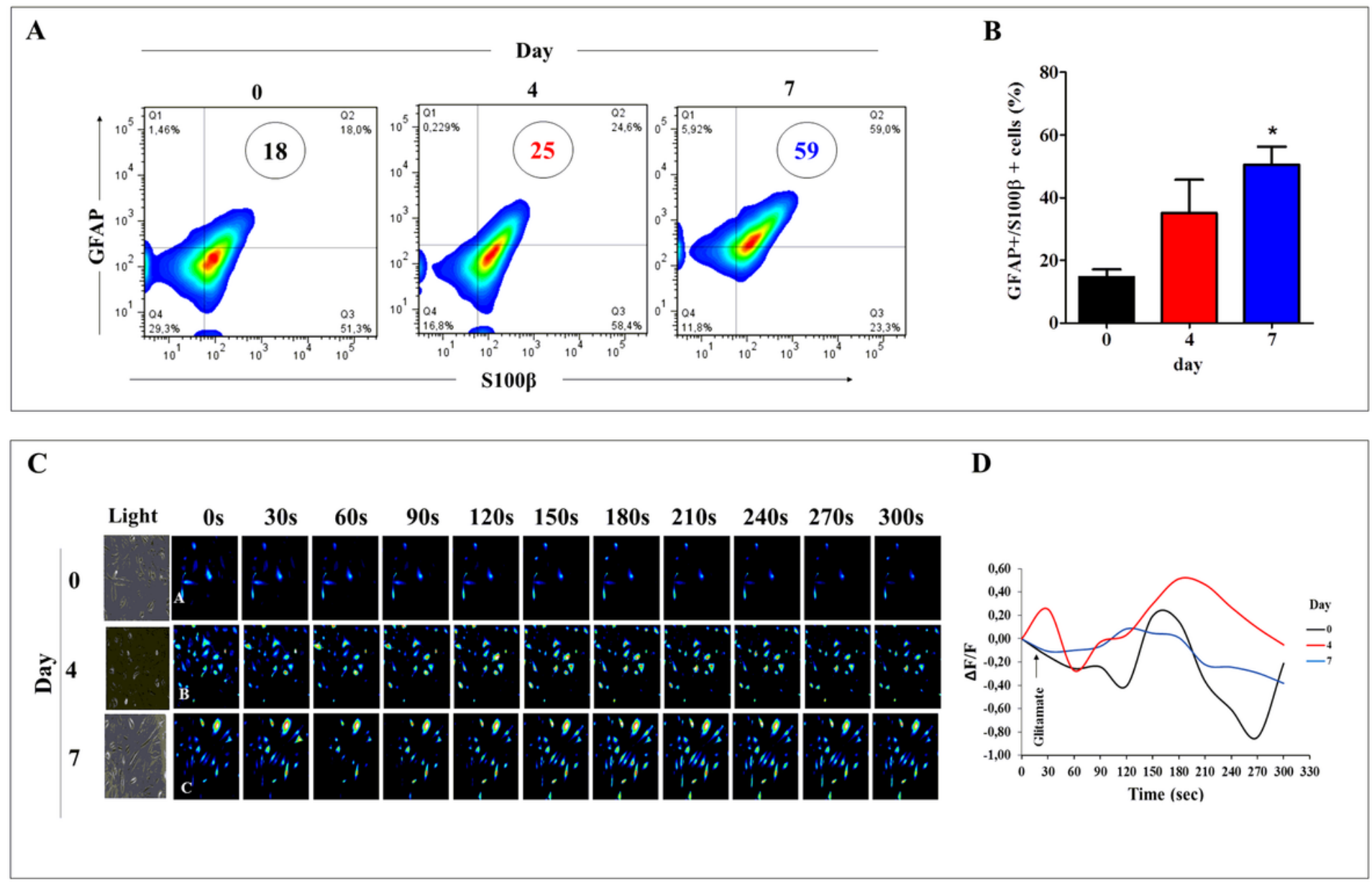

Figure 8

\section{Figure 8}

GFAP and S100ß percentage expression glutamate upregulation of cytoplasmic Ca2+ concentration in MenSCs differentiated into Astrocyte-like cells. MenSCs were cultured in Astrocyte medium as described in Materials and Methods section for 0, 4 and 7 days. Representative density plot figures showing the GFAP/ S100 $\beta$ double positive population of Astrocyte medium cultured MenSCs at day 0, 4 and 7 (A). (B) Percentage of GFAP/ S100 $\beta$ double positive cells. (C) Time-lapse images $(0,30,60,90,120,150,180$, 210, 240, 270 and 300 s) of Ca2+ fluorescence in differentiating MenSC at days 0, 4 and 7 ( $n=30$ cells imaged, $\mathrm{N}=3$ dishes) as a response to glutamate treatment. glutamate was puffed into the culture at $0 \mathrm{~s}$ (arrow). Then, Ca2+ fluorescence of cells was monitored at indicated times. Color contrast indicates fluorescence intensity: dark blue < light blue < green < yellow < red. (D) Normalized mean fluorescence signal $(\Delta F / F)$ over time, indicating temporal cytoplasmic Ca2+ elevation in response to glutamate treatment. Data are presented as means \pm SD. ${ }^{*} p<0.05 ;{ }^{* \star} p<0.01$. Image magnification $200 x$. 


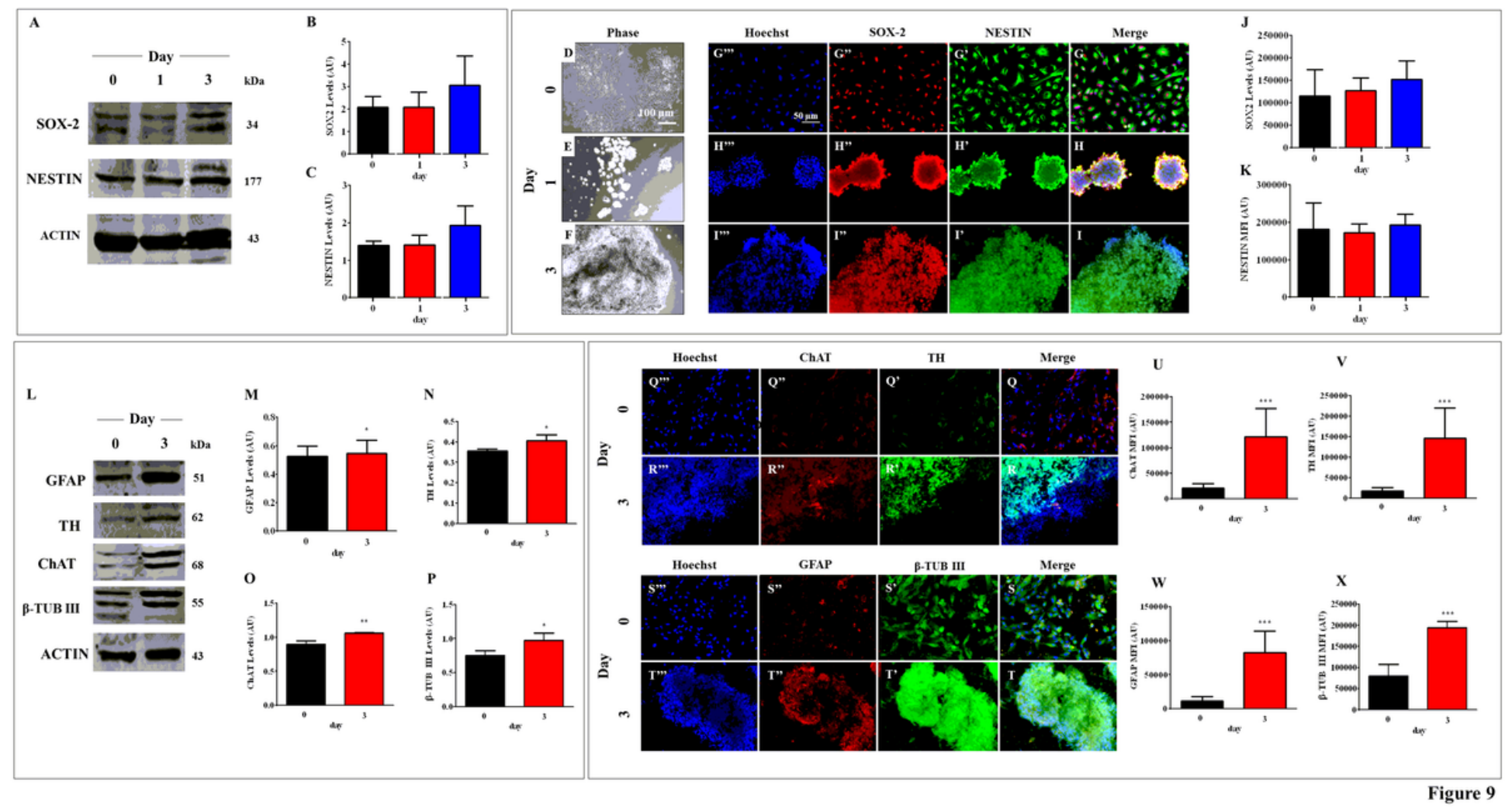

\section{Figure 9}

Western blot and immunofluorescence analysis of neurospheres/spheroids obtained from MenSCs. MenSCs were cultured in Fast-N-spheres medium as described in the Materials and Methods section for 0,1 , and 3 days. After this time, the proteins in the extracts were blotted with primary antibodies against SOX2, NESTIN, and actin proteins. The intensities of the western blot bands shown in (A) were measured $(B, C)$ by an infrared imaging system (Odyssey, LI-COR), and the intensity was normalized to that of actin. (D, E, and F) Phase-contrast light images of adherent or floating 3D spheres at day 0, 1, and 3. (G-I) Cells were double-stained as indicated in the figure with primary antibodies against SOX2 (red; G"-I") and NESTIN (green; G'-I'). The nuclei were stained with Hoechst 33342 (blue; G'--I"). (J-K) Mean Fluorescence Intensity (MFI) quantification of images obtained by immunofluorescence analysis. Undifferentiated MenSCs ( 0 days) and 3D spheroid structures (3 days) were collected and the proteins in the extracts were blotted with primary antibodies against GFAP, TH, ChAT, $\beta$-TUB III, and actin proteins. The intensities of the western blot bands shown in $(L)$ were measured $(M, N, O$, and $P)$ by an infrared imaging system (Odyssey, LI-COR), and the intensity was normalized to that of actin. (Q-T) Cells were double-stained as indicated in the figure with primary antibodies against ChAT (red; Q"-R") and TH (green; Q'-R') or GFAP (red; $S^{\prime \prime}-T^{\prime \prime}$ ) and $\beta$-TUB III (green; $S^{\prime}-T^{\prime}$ ). The nuclei were stained with Hoechst 33342 (blue; Q'"-T"'). (U-X) Mean Fluorescence Intensity (MFI) quantification of images obtained by immunofluorescence analysis. Data are presented as means $\pm S D$. ${ }^{\star} p<0.05 ;{ }^{*} p<0.01 ;{ }^{* \star} p<0.001$. Image magnification $200 x$. 


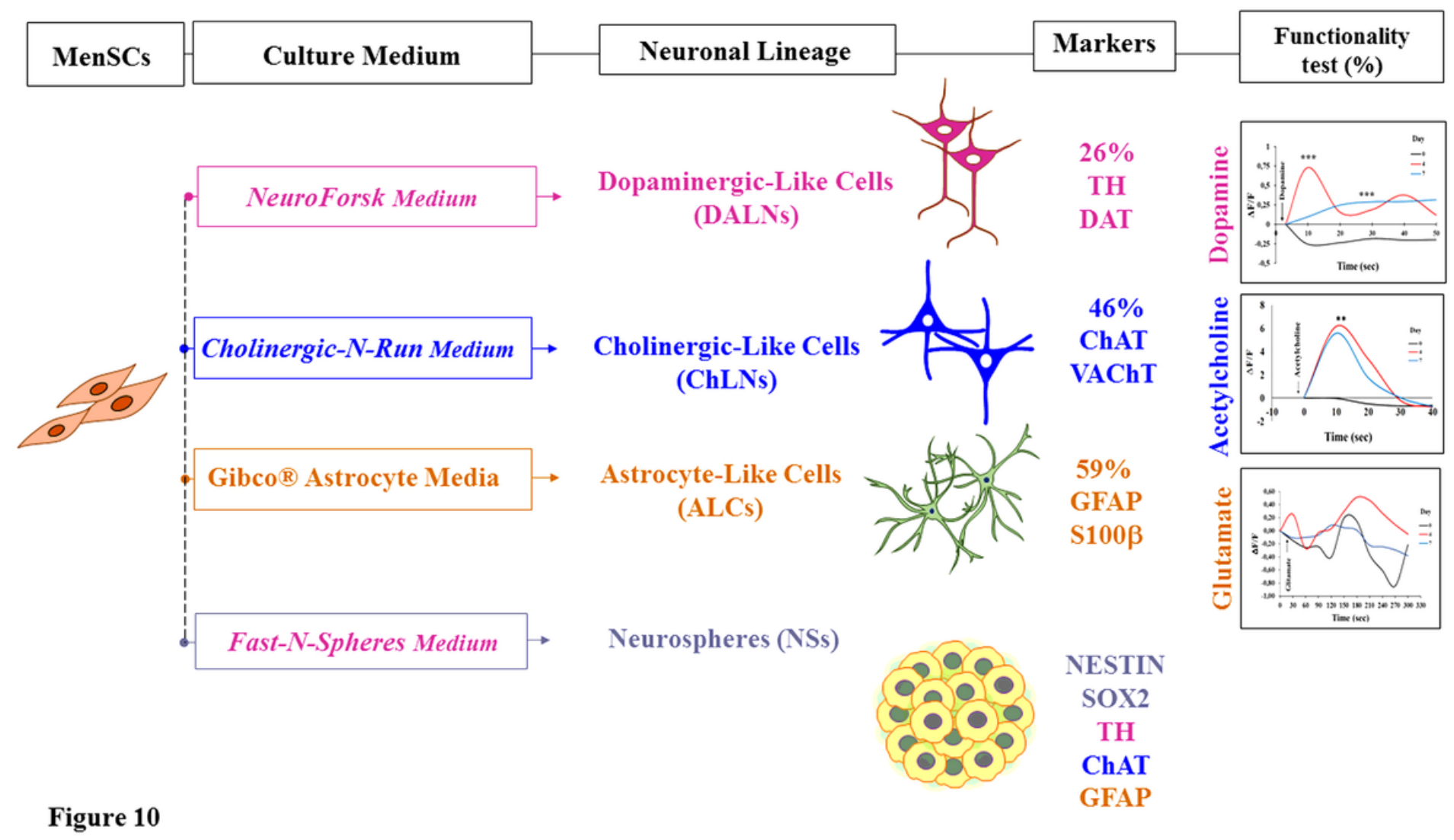

\section{Figure 10}

Human menstrual blood-derived mesenchymal stromal cells (MenSCs) readily transdifferentiate into functional dopaminergic-, cholinergic-, and astrocytic-like neuronal lineages and in neurospheres under specific in vitro culture conditions. 